\title{
Size dependent nanoindentation of a soft film on a hard substrate
}

\author{
Shaohua Chen *, Lei Liu, Tzuchiang Wang \\ LNM, Institute of Mechanics, Chinese Academy of Sciences, No. 15, Beisihuan Xilu, Beijing 100080, China
}

Received 21 August 2003; received in revised form 21 October 2003; accepted 28 October 2003

\begin{abstract}
Nanoindentation experiments on Al/glass systems show that, as the indentation depth increases, the hardness decreases during a shallow indentation, and increases when the indenter tip approaches the film-substrate interface. We associate the rise in hardness during two stages with the strong strain gradient effects, the first stage is related with the small scale effects and the second stage with the strain gradient between the indenter and the hard substrate. Using the strain gradient theory proposed by Chen and Wang and the classical plasticity theory, the observed nanoindentation behavior is modeled and analyzed by means of the finite element method, and it is found that the classical plasticity cannot explain the experiment results but the strain gradient theory can describe the experiment data at both shallow and deep indentation depths very well. The results prove that both the strain gradient effects and substrate effects exist in the nanoindentation of the film-substrate system.
\end{abstract}

(C) 2003 Acta Materialia Inc. Published by Elsevier Ltd. All rights reserved.

Keywords: Nanoindentation; Hardness; Film-substrate system; Strain gradient effect; Substrate effect

\section{Introduction}

The indentation hardness of a ductile metal is usually considered to be a measure of its yield stress in compression. According to the conventional plasticity theory, in which material properties are independent of the length scale, the measured hardness values should be independent of the indentation size. Recently, hardness has been shown to be size-dependent when the width of impression is below some limit, say about $50 \mu \mathrm{m}$ [1-4]. The measured hardness may double or even triple as the size of indent decreases from about 50 to $1 \mu \mathrm{m}$. In fact, the smaller the size of indent, the stronger the solid appears. Similar size effects have been observed for a wide range of plasticity phenomena. For example, in a thin-wire torsion and micro-thin beam bending it is shown that materials display strong size effects when the characteristic length scale is on the order of microns $[5,6]$. The strength of a particle-reinforced metal matrix composite increases with decreasing particle diameter at a fixed volume fraction of particle [7].

\footnotetext{
${ }^{*}$ Corresponding author. Tel.: +86-10-62545533-3023; fax: +86-1062561284

E-mail address: chenshaohua72@hotmail.com (S. Chen).
}

The conventional plasticity cannot predict any of those microstructural effects because no length scales are contained in it. Recent developments of strain gradient theories of plasticity have combined the microstructural and continuum descriptions of plasticity together [8-19], in which the material length scales are explicitly contained and the flow stress depends not only on the strain at a particular point, as in the conventional plasticity theory, but also on the gradients of strain at that point.

Indentation problems were investigated with several strain gradient theories. Shu and Fleck [20] failed to explain the indentation behavior using the strain gradient theory proposed by Fleck and Hutchinson, which only considered the rotation component of the strain gradient [8]. Begley and Hutchinson [21] used the theory proposed by Fleck and Hutchinson in 1997 [9] to determine the effect of the material length scale on the predicted hardness for small indents. Huang et al. [22] used mechanism-based strain gradient (MSG) theory to analyze this kind of problem and successfully explained a linear dependence of the square of the hardness, $H^{2}$, on the inverse of the indentation depth, $1 / h$, i.e. $H^{2} \propto 1 / h$. Saha et al. [23] used the MSG theory to investigate the strain gradient effects in nanoindentation 
of the film-substrate system and found that the MSG theory could describe the new phenomena well. Chen and Wang [24] successfully explained the experimental results of microindentation for the polycrystalline copper and single-crystal copper using the strain gradient theory proposed in $[18,19]$.

In the present paper, nanoindentation experiments on Al/glass systems have been carried out. Small scale effects have been found at a narrow indent depth and strain gradient effects are also found between the indenter and the substrate. The strain gradient theory proposed by Chen and Wang [18,19] is used to analyze the present experiment data, which has successfully explained the cleavage fracture in homogeneous material [19] and bimaterial [25], the size effect in particle-reinforced metal matrix composites [26], thin-wire torsion and ultra-thin beam bending $[16,17]$. In the present paper, it is found finally that the strain gradient theory can describe not only the decreasing hardness with the increasing depth of indentation for shallow indentation, which can be observed in bulk materials, but also the increase in hardness when the indenter tip approaches the film-substrate interface. The results in the present paper show that the strain gradient effects do exist in nanoindentation for a film-substrate system, and the strain gradient theory proposed by Chen and Wang $[18,19]$ can describe the new kind of strain gradient plasticity experiments.

Nanoindentation experiments and the experiment results will be shown in Section 2. The strain gradient theory used in the present paper is summarized in Section 3. In Section 4, the finite element method and the indentation model are given and the comparison of the numerical results and the experiment data is shown in Section 5. Conclusions are given in Section 6.

\section{Nanoindentation experiment}

The present nanoindentation experiments are motivated by the work of Saha et al. [23], in which nanoindentation on micro-meter film-substrate systems was studied and it was found that the new kind of strain gradient effects was different in part from the size effects in bulk materials. In the present paper, we choose to study the nanoindentation behavior of aluminum film on glass substrate with the aluminum film thickness in submicron meters.

Al films are prepared on glass substrates by the sputtering method and the base pressure in the chamber prior to sputtering is $5 \times 10^{-7}$ Torr. The sputtering pressure is 1.0 Pa. The nominal thicknesses of $\mathrm{Al}$ films are 244.7 and $850.9 \mathrm{~nm}$, respectively. The deposition rate is $160 \AA / \mathrm{min}$ and the sputtering power is $1000 \mathrm{~W}$.

The mechanical properties of the substrates and films are characterized using Nanoindentation XP II with a
Berkovich indenter. Continuous stiffness mode (CSM) is used in all experiments. The indentations are under a constant nominal strain rate $0.05 \mathrm{~s}^{-1}$. Five indentations are made in each sample and the average of these indentations is taken. Hardness and Young's modulus are firstly determined by means of the Oliver and Pharr analysis method [27]. It should be noted that Oliver and Pharr's method is for monolithic materials and only for sink-in with a fixed empirical formula. Pile-up will be produced in some materials and the degree of sink-in for different materials is different, however, it is a standard method of analysis for nanoindentation and is frequently used for thin film studies. In order to avoid the calculation of the contact area, which sometimes causes some deviations in the results due to sink-in or pile-up, especially because the film-substrate system in the present paper is made up of soft films and hard substrates, and extensive pile-up of aluminum material on the sides of the indenter is to be expected, as is proved by Tsui and Pharr [28] and Saha et al. [23,29]. In the present paper, we will use the method given by Joslin and Oliver [30], which avoids the contact area calculation and only use the loading and the unloading stiffness, to analyze the experiment data. Aluminum film and glass substrate are chosen and constant Young's modulus assumption is made because the Joslin and Oliver method can only be used for the elastically homogeneous materials and aluminum has Young's modulus close to that of the glass material.

With depth-sensing nanoindentation devices, elastic modulus is determined [31] from

$$
\begin{aligned}
& E_{\mathrm{r}}=\frac{\sqrt{\pi}}{2 \beta} S \frac{1}{\sqrt{A}}=\frac{\sqrt{\pi}}{2 \beta} \frac{\mathrm{d} P}{\mathrm{~d} h} \frac{1}{\sqrt{A}}, \\
& S=\frac{2 \beta}{\sqrt{\pi}} \sqrt{A} E_{\mathrm{r}},
\end{aligned}
$$

where $A$ is the projected area of the contact, $\beta$ is a constant that depends on the geometry of the indenter. $S=\mathrm{d} P / \mathrm{d} h$ is the slope of the load-displacement curve at the beginning of the unloading and $E_{\mathrm{r}}$ is the reduced Young's modulus.

If the Young's modulus of the film is close to that of the substrate, i.e. $E_{\mathrm{f}} \approx E_{\mathrm{s}}$, then the reduced Young's modulus can be written as

$\frac{1}{E_{\mathrm{r}}}=\frac{1-v_{\mathrm{i}}^{2}}{E_{\mathrm{i}}}+\frac{1-v_{\mathrm{f}}^{2}}{E_{\mathrm{f}}}$,

where $E_{\mathrm{i}}, E_{\mathrm{f}}$ are Young's moduli of the indenter and the film, respectively. $v_{\mathrm{i}}, v_{\mathrm{f}}$ are Poisson's ratios of the indenter and the film, respectively.

Hardness is usually defined as

$H=\frac{P}{A}$. 
Eliminating the contact area from Eqs. (1) and (4), the composite hardness for the film-substrate system is obtained as

$H=\frac{4 \beta^{2}}{\pi} \frac{P}{S^{2}} E_{\mathrm{r}}^{2}$.

If the film has a Young's modulus close to that of the substrate, the reduced modulus, $E_{\mathrm{r}}$ will approximately be a constant, which is called the constant Young's modulus assumption.

The method proposed by Joslin and Oliver [30], Eq. (5), is used in the present paper. The Young's modulus of glass is measured to be $88.5 \mathrm{Gpa}$ by nanoindentation and the measured Young's modulus of Al/glass system keeps a constant, which means that the film has a Young's modulus close to that of the substrate, we take the Young's modulus of $\mathrm{Al}$ as $E_{\mathrm{Al}}=E_{\text {glass }}=88.5 \mathrm{Gpa}$. During the test, Poisson's ratios are taken as $v_{\mathrm{Al}}=v_{\text {glass }}=0.3$. The Young's modulus of the Berkovich indenter is taken as $1140 \mathrm{Gpa}$ and the Poisson's ratio is 0.07 , as is found from literatures. The constant $\beta$ is taken as 1.034 , corresponding to the Berkovich indenter geometry. According to Eq. (5), the hardness is determined from the load, $P$, and the contact stiffness, $S$, through the parameter, $P / S^{2}$. Fig. 1 shows the composite hardness as a function of the normalized indentation depth for Al/glass systems with two different film thicknesses. From Fig. 1, one can see that at a very small indentation depth, the composite hardness decreases as the indentation depth increases, which is due to the size effects as expected on the basis of the indentation in the bulk material. With the indentation getting deeper, the hardness obtained tends to a constant value of about 1.1 Gpa for the $244.7 \mathrm{~nm}$ film and $0.9 \mathrm{Gpa}$ for the $850.9 \mathrm{~nm}$ film. The hardness is approximately constant when the indentation depth is about 0.6 times the film thickness. Then the hardness starts to increase with increasing indentation depth, a phenomenon that cannot

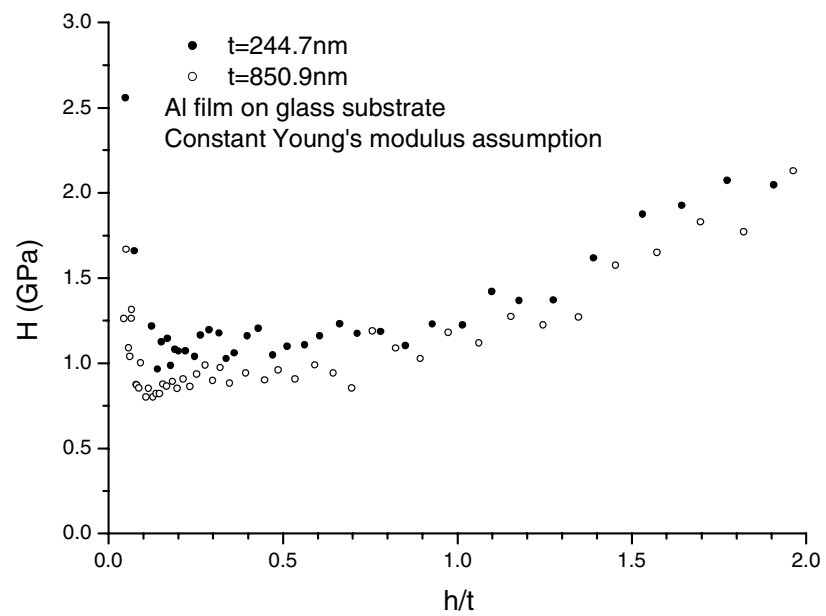

Fig. 1. Hardness versus the normalized indentation depth for two kinds of Al films on glass substrates using Joslin and Oliver method. be found in bulk materials, and may be caused by hardening related with strong gradients produced between the indenter and the substrate. When the indentation depth gets to the film-substrate interface, a more significant increase in hardness with the increasing indentation depth can be found, which is mainly due to the indenter penetration into the harder substrate.

\section{Brief review of strain gradient theory}

In order to explain the size effects in film-substrate nanoindentation experiments, the strain gradient theory proposed by Chen and Wang $[18,19]$ will be used since the classical plasticity theory does not contain intrinsic material lengths and cannot predict the effects related with the material size. The strain gradient theory is briefly reviewed as follows. It preserves the basic structure of the general couple stress theory and involves no higher-order stress or higher-order strain rates. Its key features are that the rotation gradient influences the material behavior through the interaction between Cauchy stresses and couple stresses, while the stretch gradient explicitly enters the constitutive relations through the instantaneous tangent modulus. The tangent hardening modulus is influenced by not only the generalized effective strain but also the effective stretch gradient.

In a Cartesian reference frame $x_{i}$, the strain tensor $\varepsilon_{i j}$ and the stretch gradient tensor $\eta_{i j k}$ [32] are related to the displacement $u_{i}$ by

$\varepsilon_{i j}=\frac{1}{2}\left(u_{i, j}+u_{j, i}\right) \quad \eta_{i j k}=u_{k, i j}$.

The rotation gradient is related with the independent micro-rotation vectors $\omega$ as

$\chi_{i j}=\omega_{i, j}$.

The effective strain, effective rotation gradient and effective stretch gradient are defined as

$\varepsilon_{\mathrm{e}}=\sqrt{\frac{2}{3} \varepsilon_{i j}^{\prime} \varepsilon_{i j}^{\prime}}, \quad \chi_{\mathrm{e}}=\sqrt{\frac{2}{3} \chi_{i j}^{\prime} \chi_{i j}^{\prime}}, \quad \eta_{1}=\sqrt{\eta_{i j k}^{(1)} \eta_{i j k}^{(1)}}$,

where $\varepsilon_{i j}^{\prime}, \chi_{i j}^{\prime}$ are their derivative parts and the definition of $\eta_{i j k}^{(1)}$ can be found in [32].

The constitutive relations are as follows

$$
\begin{aligned}
& \sigma_{i j}=\frac{2 \Sigma_{\mathrm{e}}}{3 E_{\mathrm{e}}} \varepsilon_{i j}^{\prime}+K \varepsilon_{m} \delta_{i j}, \\
& m_{i j}=\frac{2 \Sigma_{\mathrm{e}}}{3 E_{\mathrm{e}}} l_{\mathrm{cs}}^{2} \chi_{i j}^{\prime}+K_{1} l_{\mathrm{cs}}^{2} \chi_{m} \delta_{i j}, \\
& \begin{cases}E_{\mathrm{e}}^{2}=\varepsilon_{\mathrm{e}}^{2}+l_{\mathrm{cs}}^{2} \chi_{\mathrm{e}}^{2}, & \Sigma_{\mathrm{e}}=\left(\sigma_{\mathrm{e}}^{2}+l_{\mathrm{cs}}^{-2} m_{\mathrm{e}}^{2}\right)^{1 / 2}, \\
\sigma_{\mathrm{e}}^{2}=\frac{3}{2} s_{i j} s_{i j}, & m_{\mathrm{e}}^{2}=\frac{3}{2} m_{i j}^{\prime} m_{i j}^{\prime},\end{cases}
\end{aligned}
$$

where $E_{\mathrm{e}}$ is called the effective generalized strain and $\Sigma_{\mathrm{e}}$ is the work conjugate of $E_{\mathrm{e}} ; l_{\mathrm{cs}}$ is an intrinsic material 
length, which reflects the effects of the rotation gradient on the material behaviors; $K$ is the volumetric modulus and $K_{1}$ is the bend-torsion volumetric modulus.

In order to consider the influence of the stretch gradient, a new hardening law [16] is introduced

$$
\begin{cases}\dot{\Sigma}_{\mathrm{e}}=A^{\prime}\left(E_{\mathrm{e}}\right)\left(1+\frac{l_{1} \eta_{1}}{E_{\mathrm{e}}}\right)^{1 / 2} \dot{E}_{\mathrm{e}}=B\left(E_{\mathrm{e}}, l_{1} \eta_{1}\right) \dot{E}_{\mathrm{e}}, & \Sigma_{\mathrm{e}} \geqslant \sigma_{\mathrm{Y}}, \\ \dot{\Sigma}_{\mathrm{e}}=3 \mu \dot{E}_{\mathrm{e}}, & \Sigma_{\mathrm{e}}<\sigma_{\mathrm{Y}},\end{cases}
$$

where $B\left(E_{\mathrm{e}}, l_{1} \eta_{1}\right)$ is the hardening function; $l_{1}$ is the second intrinsic material length associated with the stretch gradient, $\sigma_{\mathrm{Y}}$ is the yield stress and $\mu$ the shear modulus.

\section{Finite element method and model}

\subsection{Choice of elements}

It is found that the choice of elements for gradient plasticity is complicated and in particular, quite sensitive to details of the constitutive relation. Xia and Hutchinson [33] discussed some choices of finite elements for strain gradient plasticity with the emphasis on plane strain cracks. Several elements have been developed for the phenomenological theory of strain gradient plasticity to investigate the crack tip field, microindentation experiments and stress concentrations around a hole. A review of these elements can be found in the paper written by Shu et al. [34].

In order to consider the strain gradient, a secondorder element can be used, such as nine-node elements. We will use nine-node elements to study the nanoindentation in the present paper. This element is only suitable for solids with vanishing higher-order stress traction on the surface. For example, the element works very well in the fracture analysis of strain gradient plasticity $[19,24,35]$, where the higher-order stress tractions vanish on the crack face and on the remote boundary. This element also works well in the study of microindentation experiments [22] because the higher-order stress tractions are zero on the indented surface. Since the strain gradient theory proposed by Chen and Wang $[18,19]$ does not include higher-order stress or higher-order stress tractions, it will work well in the present study as discussed in the following section.

\subsection{Calculation model}

The first assumption made in the present calculation is that the indenter is axis-symmetric, which greatly simplifies the finite element analysis. Furthermore, we consider a conical indenter shown in Fig. 2. The half-

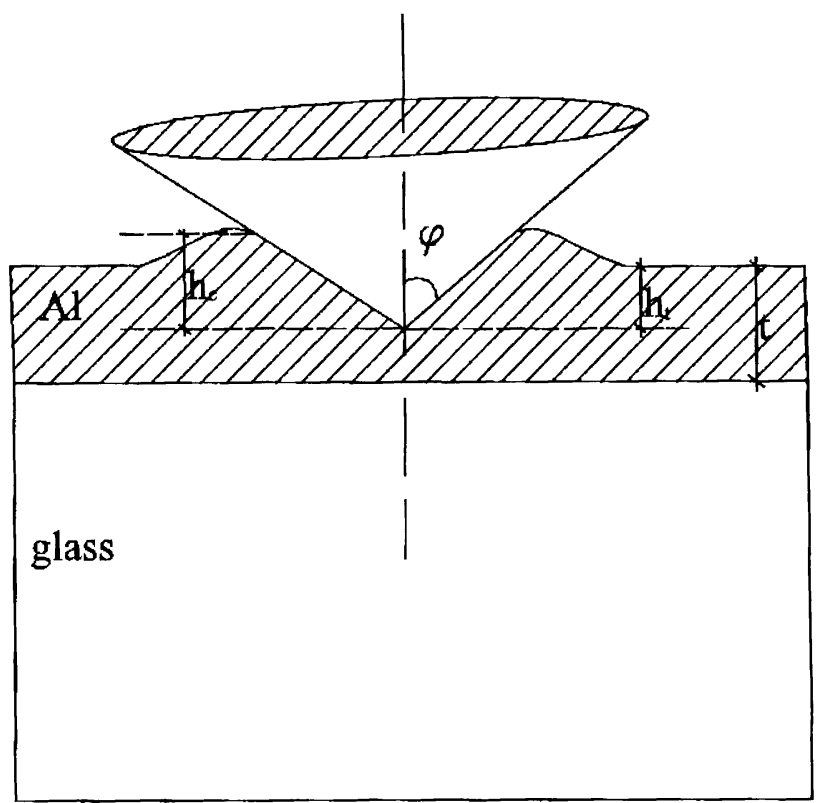

Fig. 2. Diagram of the indentation model of a soft film on a hard substrate, in which pile-up, the total indentation depth, the contact depth, contact radius are shown.

angle of the indenter is taken to be $\varphi=70.3^{\circ}$, corresponding to a Berkovich indenter. The displacement at the tip of indentation is $h_{\mathrm{t}}$, whereas the contact radius of the indentation is $a$ and the contact depth is $h_{\mathrm{c}}$. Secondly, we assume that the indenter is frictionless such that there is no sticking between the indenter and the substrate.

For a frictionless indenter, the materials on the indenter are constrained to slide up or down the face of the indenter only. For the small deformation theory and the shallow indentation in Fig. 2, the boundary conditions can be approximated by specifying the download displacement in the following and allowing the radial displacement to be free,

$u_{z}(r)=-h_{\mathrm{t}}+\frac{r}{\tan \varphi}, \quad 0 \leqslant r \leqslant a$,

where $(r, z)$ are cylindrical coordinates. No rotation vectors are imposed on the boundary and the corresponding couple stress vanishes on the free surface outside the indenter as well as on the outer boundary in the finite element analysis.

The contact between the indenter and the substrate is simulated by assuming a contact radius, $a$, and the proper indentation depth, $h_{\mathrm{t}}$, for that size of indent is found by iteration. The proper indentation depth is defined as the depth at which the normal pressure between the indenter and material goes to zero at the edge of contact, i.e. at $r=a$. Using small strain theory for shallow indenters, the pressure is given by the traction in the vertical direction. The pressure under the indenter is $\sigma_{z z}$. The correct depth is found when the nodal forces go 
to zero at the edge of contact. Since the nodal forces represent the integrated average of the tractions over the element faces, this is consistent with the zero-traction criterion. Similar method is considered in [20-23]. by

The contact depth, $h_{\mathrm{c}}$, as shown in Fig. 2 can be given

$h_{\mathrm{c}}=\frac{a}{\tan \varphi}$.

The total force, $P$, exerted on the indenter is the sum of the nodal forces in the $z$ direction of the nodes contacted with the indenter, i.e. the nodes in the region of $0 \leqslant r \leqslant a$.

The indentation hardness in the present paper is defined as

$H=\frac{P}{\pi a^{2}}$.

Since the finite element model is the same as those in [23], pile-up or sink-in is a natural result of the indentation analysis. The contact depth, $h_{\mathrm{c}}$, comparing with the total depth, $h_{\mathrm{t}}$, could be used to determine the pileup or sink-in phenomena as follows

$h_{\mathrm{c}}>h_{\mathrm{t}}$, pile-up,

$h_{\mathrm{c}}<h_{\mathrm{t}}, \quad$ sink-in.

\subsection{Material model for film-substrate system}

From experiment results, we obtain the Young's modulus of glass, $E_{\text {glass }}=88.5 \mathrm{Gpa}$. The Young's modulus of aluminum is close to that of glass and we take the Young's modulus of aluminum as $E_{\mathrm{Al}}=88.5$ Gpa in the paper. The Poisson's ratios of aluminum and glass are taken as $v_{\mathrm{Al}}=v_{\text {glass }}=0.3$ in the experiment. The yield stress of glass is very large and in the finite element calculation we regard the glass as an elastic solid, which is similar to the idea of Saha et al. [23]. The uniaxial stress-strain constitutive relation of aluminum is taken as a power law hardening form as follows

$\begin{cases}\sigma=E_{\mathrm{Al}} \varepsilon, & \sigma \leqslant \sigma_{\mathrm{Y}}, \\ \sigma=\sigma_{0} \varepsilon^{n}, & \sigma \geqslant \sigma_{\mathrm{Y}},\end{cases}$

where $\sigma_{\mathrm{Y}}$ is the yield stress of aluminum film, $n$ is the strain hardening exponent, which is related to the microstructure of films and $\sigma_{0}$ is the reference stress. As pointed in [23,36,37], the yield stress of aluminum is related to the film thickness by

$\sigma_{\mathrm{Y}}=\sigma_{\text {bulk }}+\frac{m_{\text {thick }}}{t}$.

\section{Comparison with the experiment results}

As mentioned above, the glass is regarded as an elastic solid and the aluminum film is an elastic-plastic solid. Thus the strain gradient effect exists in aluminum film when the indentation depth is very shallow, where the substrate effect does not emerge. The hardness is expected to increase when the indentation depth decreases at the shallow depth. When the indentation depth increases the substrate will influence the hardness little by little and the strain gradient between the indenter tip and the substrate will also influence the hardness significantly, which will cause the hardness to increase when the indentation depth increases.

In the numerical calculations, two film thicknesses are considered, that is, 244.7 and $850.9 \mathrm{~nm}$ for aluminum films and the thickness of substrate is assumed to be much larger than that of films. The yield stress of the bulk aluminum material is taken as $60 \mathrm{Mpa}$, i.e. $\sigma_{\text {bulk }}=60 \mathrm{Mpa}$. The thickness related parameter, $m_{\text {thick}}$, is taken as $60 \mathrm{Mpa}$ for $850.9 \mathrm{~nm}$ thickness aluminum film and $30 \mathrm{Mpa}$ for $244.7 \mathrm{~nm}$ thickness aluminum film. AFM images show that the microstructures of two thickness films are different and the grain size of 850.9 $\mathrm{nm}$ film is a little larger than that of $244.7 \mathrm{~nm}$ film, thus we take the strain-hardening exponent as 0.1 for 850.9 $\mathrm{nm}$ film and 0.05 for $244.7 \mathrm{~nm}$ film.

The experiment hardness data shown in Fig. 1 for $850.9 \mathrm{~nm}$ thickness aluminum film are also given in Fig. 3 for comparison. It is observed that the hardness predicted by the strain gradient theory in $[18,19]$ agrees very well with the experiment results over the entire depth $h \leqslant t$, even in the region of $h>t$, the numerical results agree quite well with the experiment results. For comparison, the numerical results calculated using the classical plasticity theory with the same strain hardening exponent and the yield stress for $850.9 \mathrm{~nm}$ film are also shown in Fig. 3, from which one can see that the hardness predicted by the classical plasticity theory is below the experiment data. When the indentation is

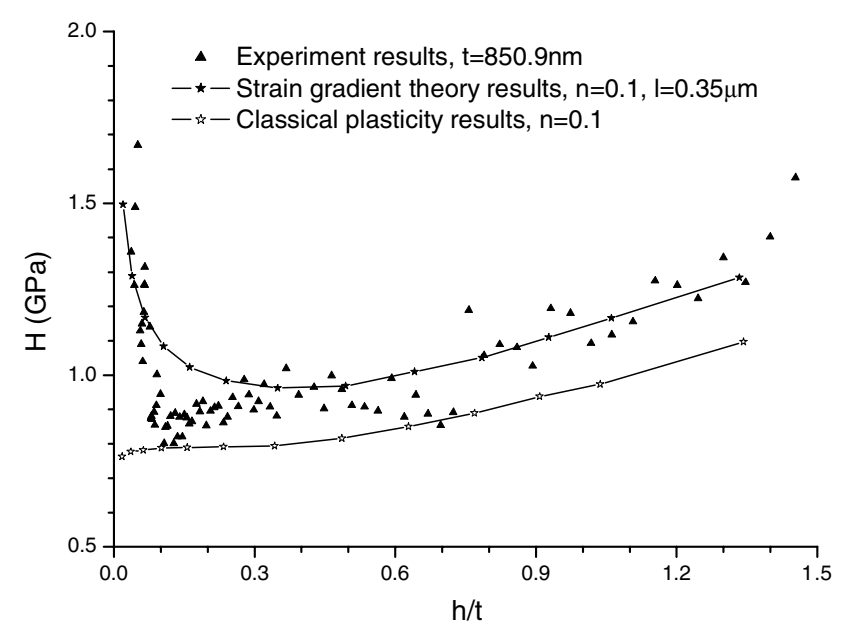

Fig. 3. The hardness versus the normalized depth for $850.9 \mathrm{~nm} \mathrm{Al} \mathrm{film}$ on a glass substrate with the experiment data and numerical results for both strain gradient theory and the classical plasticity theory. 
shallow, the hardness predicted by the classical plasticity theory almost keeps a constant and no increase in hardness can be observed. When the indentation depth is comparable to the film thickness, the hardness can be observed to increase due to the substrate effect but the hardness value is smaller than the experiment data, which demonstrates that the strain gradient effects exist between the indenter tip and the substrate and cause an increased hardness. Fig. 3 shows that in the nanoindentation processing on film-substrate system, strain gradient effects are important not only at the shallow indentation depth but also at the deep depth, where the indentation depth is comparable to the film thickness. From comparison of the numerical calculation and the experiment data, the intrinsic length for $850.9 \mathrm{~nm}$ thickness aluminum film is $l_{1}=0.35 \mu \mathrm{m}$, which lies in the region of $0.1-10 \mu \mathrm{m}$.

The experiment data for $244.7 \mathrm{~nm}$ thickness aluminum film and the numerical calculation results are shown in Fig. 4 for the hardness versus the normalized indentation depth. From Fig. 4, one can see that the strain gradient results agree well with the experiment data while the results predicted by the classical plasticity theory fall below the experiment data and at the shallow indentation depth, the hardness predicted by the classical theory keeps a constant and then increases at some indentation depth, which is caused by the substrate effects. From the comparison between the numerical calculation results and the experiment data, the intrinsic length for the aluminum film of $244.7 \mathrm{~nm}$ in thickness is $0.3 \mu \mathrm{m}$, i.e. $l_{1}=0.3 \mu \mathrm{m}$.

Numerical calculation results show that pile-up emerges more obviously in the deformed finite element mesh as the indentation depth increases, which could cause significant deviations of experiment hardness data measured if the pile-up effect is not considered.

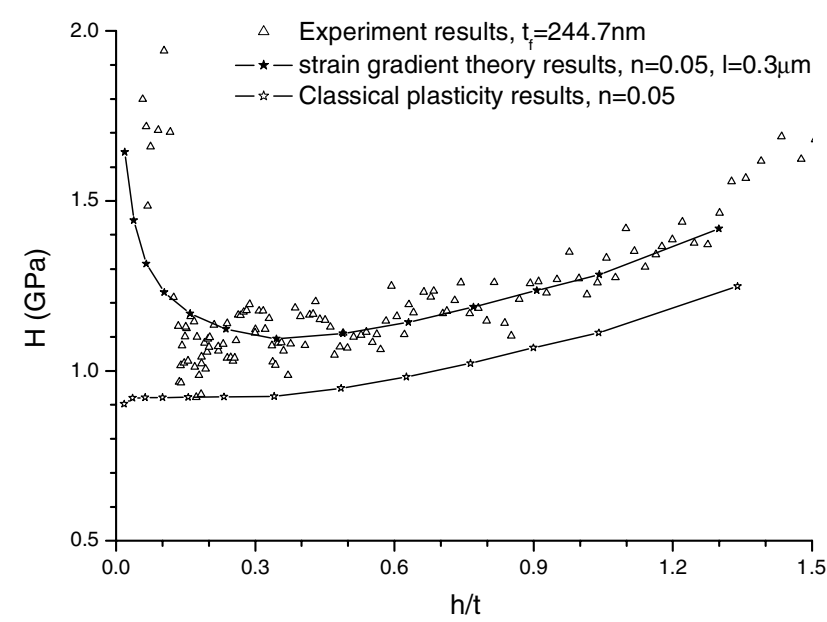

Fig. 4. The hardness versus the normalized depth for $244.7 \mathrm{~nm} \mathrm{Al} \mathrm{film}$ on a glass substrate with the experiment data and numerical results for both strain gradient theory and the classical plasticity theory.

\section{Conclusions}

In the present paper, two kinds of film-substrate systems with sub-micrometer thicknesses are studied using nanoindentation experiments. The aim of the experiment is to create a strong strain gradient by indenting a soft metal film on the hard substrate to observe whether there is a strain gradient hardening phenomena in the film-substrate system. From the experiment results, strong strain gradient effects are found when the indentation is shallow compared to the film thickness, furthermore, substrate effects and strain gradient effects between the indenter and the substrate are found to influence the film-substrate hardness significantly when the indenting depth is comparable to the film thickness.

Strain gradient theory proposed by Chen and Wang $[18,19]$ is used and the numerical results can explain the experiment data very well both at the shallow indentation depth and at the depth comparable to the film thickness. Also the substrate effects can be described well during the numerical calculation using the strain gradient theory, which cannot be found in the nanoindentation on a bulk material. It demonstrates that the strain gradient theory used in the present paper can describe this new kind of phenomena.

\section{Acknowledgements}

The authors would like to express their gratitude to the National Science Foundation of China (Nos. 10202023, 10272103) and the Excellent Post-doctoral Research-Starting Fund of CAS for financial support.

\section{References}

[1] Ma Q, Clarke DR. J Mater Res 1995;10:853.

[2] McElhaney KW, Vlassak JJ, Nix WD. J Mater Res 1998;13:1300.

[3] Nix WD. Metall Trans 1989;20A:2217.

[4] Poole WJ, Ashby MF, Fleck NA. J Mech Phys Solids 1996; 44:465.

[5] Fleck NA, Muller GM, Ashby MF, Hutchinson JW. Acta Met Mater 1994;42:475.

[6] Stolken JS, Evans AG. Acta Mater 1998;46:5109.

[7] Lloyd DJ. Int Mater Rev 1994;39:1.

[8] Fleck NA, Hutchinson JW. J Mech Phys Solids 1993;41:1825.

[9] Fleck NA, Hutchinson JW. Adv Appl Mech 1997;33:295.

[10] Gao H, Huang Y, Nix WD, Hutchinson JW. J Mech Phys Solids 1999;47:1239.

[11] Huang Y, Gao H, Nix WD, Hutchinson JW. J Mech Phys Solids 2000;48:99.

[12] Gao H, Huang Y. Int J Solids Struct 2001;38:2615.

[13] Acharya A, Shawki TG. J Mech Phys Solids 1995;43:1751.

[14] Aifantis EC. Trans ASME J Eng Mater Technol 1984;106:326.

[15] Zbib H, Aifantis EC. Acta Mech 1992;92:209.

[16] Chen SH, Wang TC. Acta Mater 2000;48:3997.

[17] Chen SH, Wang TC. Int J Plasticity 2002;18:971.

[18] Chen SH, Wang TC. Eur J Mech A/Solids 2001;20:739. 
[19] Chen SH, Wang TC. Int J Solids Struct 2002;39:1241.

[20] Shu JY, Fleck NA. Int J Solids Struct 1998;35:1363.

[21] Begley MR, Hutchinson JW. J Mech Phys Solids 1998;46:2049.

[22] Huang Y, Xue Z, Gao H, Nix WD, Xia ZC. J Mater Res 2000;15:1786.

[23] Saha R, Xue ZY, Huang Y, Nix WD. J Mech Phys Solids 2001;49:1997.

[24] Chen SH, Wang TC. Acta Mech, 2003 [in press].

[25] Chen SH, Wang TC. Int J Fracture 2002;117:25.

[26] Chen SH, Wang TC. Acta Mech 2002;157:113.

[27] Oliver WC, Pharr GM. J Mater Res 1992;7:1564.
[28] Tsui TY, Pharr GM. J Mater Res 1999;14:292.

[29] Saha R, Nix WD. Acta Mater 2002;50:23.

[30] Joslin DL, Oliver WC. J Mater Res 1990;5:123.

[31] Sneddon IN. Int J Eng Sci 1965;3:47.

[32] Smyshlyaev VP, Fleck NA. J Mech Phys Solids 1996;44:465.

[33] Xia ZC, Hutchinson JW. J Mech Phys Solids 1996;44:1621.

[34] Shu JY, King WE, Fleck NA. Int J Numer Methods Eng 1999;44:373.

[35] Wei Y, Hutchinson JW. J Mech Phys Solids 1997;45:1253.

[36] Venkatraman R, Bravman JC. J Mater Res 1992;7:2040.

[37] Nix WD. Scripta Mater 1998;39:545. 